\title{
ON THE CROSSING OF EXTREMALS AT FOCAL POINTS
}

\section{J. SAVAGE}

Morse and Littauer ${ }^{1}$ have proved the following theorem for an analytic Finsler space, where $g$ is an extremal transversal to the (analytic) hypersurface $\Sigma$.

Theorem. A necessary and sufficient condition that $p$ on $g$ be a focal point of $\Sigma$ is that the family of extremals cut transversaly by $\Sigma$ near $g$ shall fail to cover the neighborhood of $p$ simply.

The purpose of the present paper is to prove this theorem on the weaker hypothesis that the Finsler space and $\Sigma$ are of class $C^{\prime \prime \prime}$.

As pointed out in M. L. the sufficiency of the condition is trivial, and in proving the necessity there is no loss in generality if we assume $p$ to be a first focal point. It is further clear from M. L. that the theorem is a consequence of the following lemma.

LEMMA I. If $p$ is a first focal point on $g$ contained in a (simply covering) field $R$ of extremals transversal to $\Sigma$, then there exists a first focal point $q$ covered by $R$ and a subfield $S$ of $R$ covering $q$ and such that the Hilbert integral is independent of path for paths confined to $S$.

Before proceeding to the proof of Lemma I we will establish a secondary lemma.

Lemma II. Let $T$ be a transformation of class $C^{\prime}$ mapping a closed coordinate neighborhood $A$ into a closed Riemannian manifold $B$, then almost all points of $B$ (in the measure theoretic sense) have finite counter images.

Proof. Call the set of points $K \subset A$ at which the Jacobian of $T$ vanishes critical points, then I assert that if the counter image $T^{-1} b$, $b \in B$, is infinite, it contains a critical point. In fact if $b^{i}$ are the coordinates of such a point $b$, there is a convergent sequence of points of $T^{-1} b$ with coordinates $a_{\sigma}^{i}$ approaching a point $a_{0}$ from a definite direction, as is expressed by the following set of equations.

$$
\begin{gathered}
a_{\sigma}^{i} \rightarrow a_{0}^{i}, \xi_{\sigma}^{i}=\left(a_{\sigma}^{i}-a_{0}^{i}\right) /\left(\Sigma_{i}\left(a_{\sigma}^{i}-a_{0}^{i}\right)^{2}\right)^{1 / 2} \rightarrow \xi_{0}^{i}, \\
T^{i}\left(a_{\sigma}^{j}\right)=b^{i}=T^{i}\left(a_{0}^{j}\right) .
\end{gathered}
$$

Received by the editors November 5, 1942.

1 Marston Morse and S. B. Littauer, A characterization of fields in the calculus of variations, Proc. Nat. Acad. Sci. U.S.A. vol. 18 (1932) pp. 724-730. This paper will hereafter be designated by M. L. 
By the theorem of the mean we have

(2) $T^{i}\left(a_{\sigma}^{j}\right)=T^{i}\left(a_{0}^{j}\right)+\Sigma_{k} \frac{\partial T^{i}\left(a_{0}^{j}+\theta_{\sigma}^{i}\left(a^{j}-a_{\sigma}^{j}\right)\right)}{\partial a^{k}}\left(a_{o}^{j}-a_{0}^{j}\right), 0 \leqq \theta_{\sigma}^{i} \leqq 1$, finally

$$
\Sigma_{k} \frac{\partial T^{i}\left(a_{0}^{j}\right)}{\partial a^{k}} \xi_{0}^{k}=0
$$

so $a_{0} \in K$.

It remains to be shown that $T K$ is of measure zero. But this follows quite easily from the measure theoretic significance of the Jacobian of $T$.

Proof of Lemma I. Let $s=\left(s_{1}, \cdots, s_{n-1}\right)$ denote coordinates of points of $\Sigma$ relative to some admissible coordinate system of $\Sigma$ covering the intersection of $g$ and $\Sigma$ at $s_{0}$, and along any geodesic transversal to $\Sigma$ in this neighborhood let $r$ denote (Finsler) length from $\Sigma$ (considered positive in the direction of $p$ ). Now according to the hypothesis of the lemma if $r_{0}$ is the arc length along $g$ from $\Sigma$ to $p$, there is a $\delta$ such that for $\left|s^{i}-s_{0}^{i}\right| \leqq \delta$ and $-\delta \leqq r \leqq r_{0}+\delta$ the numbers $(r, s)$ constitute a closed coordinate neighborhood $U$ of class $C^{0}$.

Of course the coordinate neighborhood $U$ is not admissible. In fact if $X$ denotes an admissible coordinate neighborhood of $p$ and $P$ the natural transformation from $U$ to $X$, then the fact that $p$ is a focal point is expressed by the vanishing of the Jacobian $\Delta(r, s)$ of $P$ at $r_{0}, s_{0}$.

We are assuming that $p$ is a first focal point. According to a technique of Morse ${ }^{2}$ the locus of first focal points near $p$ is a hypersurface determined by an equation of the form $r=f(s)$. Now I assert that there is a point $q$ with coordinates $r_{1}=f\left(s_{1}\right)$, such that, in a neighborhood of $q, f$ is of class $C^{\prime}$, and all focal points in that neighborhood lie on the locus $r=f(s)$. To find such a point choose $s_{1}$ in such a way as to maximize the rank of the determinant $\Delta(f(s), s)$ in the neighborhood of $s_{0}$. Call this rank $k$. By slightly sharpening a result of Morse ${ }^{3}$ we can find a $(k+1)$ minor $\Delta^{*}(r, s)$ of $\Delta(r, s)$ such that $\partial \Delta^{*}(f(s), s) / \partial r$ $\neq 0$. From the assumption that the rank of $\Delta(f(s), s)$ is at most $k$ in the neighborhood of $s_{1}$ it follows that $\Delta^{*}(f(s), s) \equiv 0$ in that neighborhood, but now the implicit function theorem applies to show that $f$ is of class $C^{\prime}$ near $s_{1}$. Finally we conclude, following Morse,$^{4}$ that all

${ }^{2} \mathrm{Cf}$. Marston Morse, The calculus of variations in the large, Amer. Math. Soc. Colloquium Publications vol. 18 (1934) p. 235 Lemma 13.1.

${ }^{3}$ Marston Morse, The order of vanishing of the determinant of a conjugate base, Proc. Nat. Acad. Sci. U.S.A. vol. 17 (1931) pp. 319-320.

${ }^{4}$ See Calculus of variations in the large, loc. cit. 
focal points in the neighborhood of $q$ lie on the locus $r=f(s)$.

We are now in a position to construct the following set-up. Let $Y$ be an admissible coordinate neighborhood containing $q$ and the extremal of $R$ joining $q$ to $\Sigma$. Let $S$ be a closed subfield of $R$, contained in $Y$, covering $q$ and such that the only focal points of $\Sigma$ within $S$ satisfy $r=f(s)$. Call this intersection of the focal point locus with $S$ $\pi$. Let $V$ denote the sub-neighborhood of $U$ covered by $S$.

Now we must consider the Hilbert integral in the region covered by $S$. This is a certain line integral $I$ to be evaluated with respect to the coordinate system $Y$. The corresponding integral $I^{*}$ with respect to $V$ turns out according to M. L. to be independent of path, and we would like to make the same conclusion about $I$. The difficulty lies in the fact that though the natural transformation $Q$ from $V$ to $Y$ is of class $C^{\prime}$, its inverse is differentiable only on the complement of $Q \pi$. We can get around this by approximating arbitrary curves of class $C^{\prime}$ in $Y \cap S$ in the derivative by rectilinear polygons (with respect to $Y$ ) which intersect $Q \pi$ in only a finite number of points. This is easily achieved by means of Lemma II, from which we can conclude that if a point is not on $Q \pi$, then almost all rays (with respect to $Y$ ) issuing from it intersect $Q \pi$ in only a finite number of points. $Q^{-1}$ carries such polygons into curves differentiable at all but a finite number of points. $I^{*}$ can be extended to these curves by means of RiemannCauchy integration. The proof of the lemma is then easily completed.

The Institute for Advanced Study and

CORNELl UNIVERSITY 